\title{
Progression of endothelial dysfunction, atherosclerosis, and arterial stiffness in stable kidney transplant patients: a pilot study
}

Joey Junarta ${ }^{1,2}$, Nina Hojs ${ }^{1}$, Robin Ramphul ${ }^{1}$, Racquel Lowe-Jones ${ }^{1}$, Juan C. Kaski ${ }^{2}$ and Debasish Banerjee ${ }^{1,2^{*}}$ (D)

\begin{abstract}
Background: Kidney transplant patients suffer from vascular abnormalities and high cardiovascular event rates, despite initial improvements post-transplantation. The nature of the progression of vascular abnormalities in the longer term is unknown. This pilot study investigated changes in vascular abnormalities over time in stable kidney transplant patients long after transplantation.

Methods: Brachial artery flow-mediated dilation (FMD), nitroglycerin-mediated dilation, carotid-femoral pulse wave velocity (cf-PWV), ankle-brachial pressure index, and common carotid artery intima-media thickness (CCA-IMT) were assessed in 18 kidney transplant patients and 17 controls at baseline and 3-6 months after.

Results: There was no difference in age (51 \pm 13 vs. $46 \pm 11 ; P=0.19)$, body mass index ( $26 \pm 5$ vs. $25 \pm 3 ; P=0.49$ ), serum cholesterol ( $4.54 \pm 0.96$ vs. $5.14 \pm 1.13 ; P=0.10)$, systolic blood pressure (BP) $(132 \pm 12$ vs. $126 \pm 12 ; P=0.13)$, diastolic BP ( $82 \pm 9$ vs. $77 \pm 8 ; P=0.10$ ), or diabetes status ( 3 vs. $0 ; P=0.08$ ) between transplant patients and controls. No difference existed in vascular markers between patients and controls at baseline. In transplant patients, FMD decreased $(-1.52 \pm 2.74 ; P=0.03)$, cf-PWV increased $(0.62 \pm 1.06 ; P=0.03)$, and CCA-IMT increased $(0.35 \pm 0.53 ; P=$ 0.02). No changes were observed in controls.

Conclusion: Markers of vascular structure and function worsen in the post-transplant period on long-term followup, which may explain the continued high cardiovascular event rates in this population.
\end{abstract}

Keywords: Endothelial function, Arterial stiffness, Atherosclerosis, Kidney transplantation

\section{Background}

Patients with chronic kidney disease (CKD) have a higher burden of cardiovascular disease (CVD), including kidney transplant recipients [1]. Although cardiovascular risk factors improve in the immediate perioperative period, the long-term risk remains high $[2,3]$. CVD is the commonest cause of death in transplant patients with a surviving graft, more so than infection or malignancy [4].

\footnotetext{
* Correspondence: debasish.banerjee@stgeorges.nhs.uk

This author takes responsibility for all aspects of the reliability and freedom from bias of the data presented and their discussed interpretation.

${ }^{1}$ Renal and Transplantation Unit, St George's University Hospitals, NHS Foundation Trust, London, UK

${ }^{2}$ Cardiology Clinical Academic Group, Molecular and Clinical Sciences

Research Institute, St George's University of London, London, UK
}

Endothelial dysfunction, arterial stiffness, and accelerated atherosclerosis are common in stable kidney transplant patients and may contribute to the high cardiovascular event rate $[3,5,6]$. Endothelial dysfunction, a prerequisite to atherosclerosis, encompasses numerous maladaptive alterations adversely affecting vascular tone, haemostasis, and inflammatory processes within the arterial wall [7]. Both traditional and nontraditional risk factors in transplant patients can induce endothelial dysfunction $[3,8]$. Calcification of the arterial wall is common in transplant patients and contributes to vascular stiffness $[3,9]$.

The nature of the changes in these vascular abnormalities in kidney transplant recipients is unknown. Previous studies mostly examined changes in the vascular 
properties of transplant patients pre-transplantation and immediately post-transplantation. They were not examined in stable transplant recipients long after transplantation. If these changes are adverse, they may be the result of novel risk factors post-transplantation and may be a cause of the high cardiovascular event rate. This pilot study investigated the changes in endothelial dysfunction, arterial stiffness, and atherosclerosis in stable kidney transplant recipients long into the posttransplant period.

\section{Methods}

\section{Study population and design}

Participants included patients recruited from the transplantation clinic, patient relatives, and staff volunteers. Patients were eligible if they were between 18 to 80 years of age with stable kidney function for $\geq 3$ months (estimated glomerular filtration rate [eGFR] change $<5 \mathrm{ml} /$ $\min / 1.73 \mathrm{~m}^{2}$ ) and have been transplanted for $\geq 6$ months with or without previous dialysis. Exclusion criteria included a history of malignancy, heart failure, vasculitis, lupus, myocardial infarction or cerebrovascular event within 6 months or recent hospitalisation within 3 months prior to starting the study. The study was approved by the London South East Research Ethics Committee. All participants gave informed consent prior to their inclusion in the study. Vascular parameters were measured in a quiet vascular laboratory with a controlled temperature of $22-24^{\circ} \mathrm{C}$ at recruitment (baseline visit) and 3-6 months after recruitment (second visit). Figure 1 demonstrates a flow chart of the study design. Vascular parameters measured include brachial flow-mediated dilation (FMD), nitroglycerin-mediated dilation (NMD), carotid-femoral pulse wave velocity (cf-PWV), and common carotid artery intima-media thickness (CCA-IMT). Participants were required to withhold taking anti- hypertensive medications $24 \mathrm{~h}$ before vascular assessment to prevent interference. In the absence of local data in transplant patients we used our previous data in CKD patients for power calculation. For pre-dialysis CKD patients in our previous study the FMD was $3.1 \pm$ $3.3 \%$ [10]. To demonstrate a clinically significant difference of $60 \%$ with $\alpha=0.05$ and $\beta=0.80,27$ patients are needed. For CKD patients (pre-dialysis and post-kidney transplantation) in our previous study the CCA-IMT was $0.77 \pm 0.17$ [3]. To demonstrate a clinically significant difference of $10 \%$ over 1 year with $\alpha=0.05$ and $\beta=$ $0.80,36$ patients are needed. Assuming a dropout rate of $10 \%, 40$ patients are needed. The main aim of this pilot study was to recruit enough patients and volunteers to demonstrate a difference in change of endothelial function.

\section{Clinical characteristics}

A standardised data form was used by researchers to record participants' medical histories, including to obtain systematic information on the presence of cardiovascular risk factors. Weight, height, waist and hip circumference, and two blood pressure (BP) measurements were taken. Body mass index (BMI) and waist to hip ratio (WHR) were calculated.

Flow-mediated dilation, nitroglycerin-mediated dilation, common carotid artery intima-media thickness, and carotid-femoral pulse wave velocity

Brachial FMD, NMD, and CCA-IMT were performed as described in previous studies by our group [3]. CfPWV was performed as described before [11]. Brachial FMD and NMD were assessed using a standard HighDefinition-Imaging-3000 ultrasound system (ATL, Bothell, WA, USA) equipped with a $12-5 \mathrm{MHz}$ lineararray transducer. Cf-PWV was assessed using the

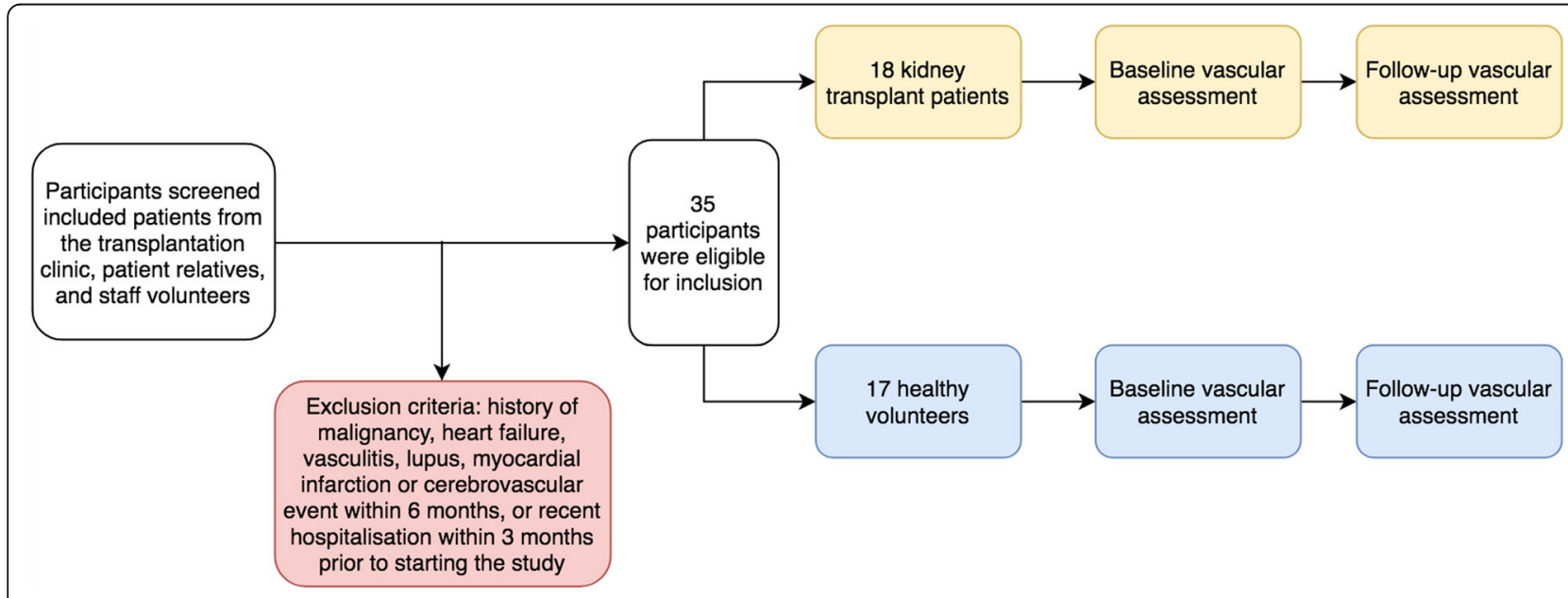

Fig. 1 Flowchart of study design 
SphygmoCor2000 system (AtCor Medical, Sydney, Australia). CCA-IMT was measured using a Vivid7 ultrasound machine (GE Healthcare, Wauwatosa, WI, USA) equipped with a linear $13-5 \mathrm{MHz}$ transducer. The overall mean (standard deviation) intra-operator variability of FMD within our department is 0.90 $(0.48) \%[12]$.

\section{Ankle-brachial pressure index}

Ankle-brachial pressure index (ABPI) was assessed with a continuous wave Doppler instrument $(8-10 \mathrm{MHz})$. The ABPI of each leg was calculated by dividing the highest systolic BP in the desired leg between the measurement of the dorsalis pedis and posterior tibial artery divided by the highest systolic BP between the two arms. The mean ABPI value for each participant was derived from the average between the ABPI of the right and left leg.

\section{Laboratory measurements}

Blood and urine samples were collected for biochemical analysis at the South West London Pathology (SWLP) services. The internal quality assurance of the SWLP service is guaranteed by strict adherence to analytical protocols and a comprehensive internal quality control programme. The SWLP service subscribes to all national external quality assessment schemes for the tests they provide and their laboratories are also registered with the Clinical Pathology Accreditation of the United Kingdom Accreditation Service, which ensures that the services meet the stringent requirements expected from a pathology service. eGFR was calculated using the Chronic Kidney Disease Epidemiology Collaboration (CKD-EPI) 2009 equation [13].

\section{Statistical analyses}

Continuous variables were analysed with an independent samples t-test, categorical variables using a chi-squared test, and within-group comparisons using a paired t-test. Bivariate correlations between continuous variables were calculated using Pearson's correlation coefficient ( $r$ ). Point-Biserial correlation $\left(\mathrm{r}_{\mathrm{pb}}\right)$ was used to determine correlations between binary and continuous variables. A two-sided $P$-value of $<0.05$ was used to determine statistical significance. Analyses were performed using SPSS version 25.0 (SPSS Inc., Chicago, IL, USA).

\section{Results}

Eighteen kidney transplant patients and 17 healthy volunteers were enrolled in the study. The mean number of days between the first and second visit for transplant patients was $161 \pm 36$ days and $185 \pm 52$ days for controls. The mean duration on dialysis (haemodialysis and/or peritoneal dialysis) before transplantation was $29 \pm 14$ months (median 29, interquartile range [IQR] 28). The median time since transplantation at recruitment was 86 months with an IQR of 123 months. Transplant patients were taking up to 3 of 6 different immunosuppressants at recruitment. The immunosuppressants used were Tacrolimus (89\%), Mycophenolate Mofetil (39\%), Prednisolone (44\%), Azathioprine (22\%) and Sirolimus (6\%). The aetiology of CKD in our transplant patients were the following: autosomal dominant polycystic kidney disease (4), IgA nephropathy (4), hypertension (2), pyelonephritis (1), focal segmental glomerulosclerosis (1), gout nephropathy (1), and type 2 diabetes mellitus (1). Four transplant patients had an unclear aetiology to their CKD.

\section{Baseline parameters in study participants}

Table 1 shows the baseline clinical characteristics and circulating biomarkers in study participants. The kidney transplant patient group had reduced eGFR, more dyslipidaemics, and elevated parathyroid hormone and Nterminal-pro-brain natriuretic peptide levels. Table 2 shows the baseline vascular structure and function of participants. No difference existed between transplant patients and controls.

\section{Changes in clinical, circulating biomarkers, and in cardiovascular structure and function in study participants}

Table 1 shows changes in clinical characteristics and circulating biomarkers in participants. In controls, systolic BP (SBP) decreased, while vitamin D and corrected calcium levels increased. In transplant patients, vitamin D levels also increased while eGFR declined. Vitamin D levels improved presumably because participants were able to request their laboratory results and may have started vitamin D therapy at their own initiative or in guidance with a clinician. Vascular structure and function did not change in controls (Table 2). In transplant patients, brachial FMD decreased $(-1.52 \pm 2.74 ; P=$ $0.03)$, while cf-PWV $(0.62 \pm 1.06 ; P=0.03)$ and CCAIMT increased $(0.35 \pm 0.53 ; P=0.02)$ (Table 2$)$.

\section{Association of changes in FMD, cf-PWV, and CIMT in kidney transplant patients}

No significant correlation existed between the decline in eGFR and changes in FMD $(r=0.21 ; P=0.42)$, cf-PWV $(r=0.30 ; P=0.23)$, or CIMT $(r=0.37 ; P=0.15)$. Changes in FMD was associated with baseline haemoglobin $(r=$ $0.52, P=0.03)$, corrected calcium $(r=0.61, P=0.01)$, and transferrin $(r=0.53, P=0.03)$. Changes in FMD were not associated with age $(r=0.43 ; P=0.08)$, BMI $(r=0.45$; $P=0.05)$, WHR $(r=0.31 ; P=0.21)$, gender $\left(\mathrm{r}_{\mathrm{pb}}=-0.18\right.$; $P=0.48), \mathrm{DM}\left(\mathrm{r}_{\mathrm{pb}}=0.18 ; P=0.47\right), \mathrm{SBP}(r=-0.32 ; P=$ $0.07)$, diastolic BP (DBP) $(r=-0.03 ; P=0.89)$, or 
Table 1 Clinical characteristics and circulating biomarkers in study participants

\begin{tabular}{|c|c|c|c|c|c|c|c|}
\hline \multirow[t]{2}{*}{ Parameter } & \multicolumn{3}{|c|}{ Healthy controls $(n=17)$} & \multicolumn{3}{|c|}{ Kidney transplant patients $(n=18)$} & \multirow{2}{*}{$\begin{array}{l}\text { Between-group comparisor } \\
\text { Baseline results } P \text {-value }\end{array}$} \\
\hline & Baseline & Second visit & $P$-value & Baseline & Second visit & $P$-value & \\
\hline Age (years) & $45.82 \pm 10.85$ & NA & NA & $51.28 \pm 13.29$ & NA & NA & 0.19 \\
\hline Gender M/F & $5 / 12$ & NA & NA & $9 / 9$ & NA & NA & 0.21 \\
\hline Body mass index $\left(\mathrm{kg} / \mathrm{m}^{2}\right)$ & $24.59 \pm 2.59$ & $25.00 \pm 2.66$ & 0.09 & $25.56 \pm 5.18$ & $24.03 \pm 7.63$ & 0.36 & 0.49 \\
\hline Waist/Hip & $0.82 \pm 0.07$ & $0.80 \pm 0.06$ & 0.10 & $0.86 \pm 0.10$ & $0.93 \pm 0.14$ & 0.07 & 0.16 \\
\hline Systolic blood pressure (mmHg) & $125.53 \pm 12.39$ & $119.76 \pm 10.62$ & 0.02 & $131.94 \pm 11.79$ & $130.44 \pm 10.20$ & 0.61 & 0.13 \\
\hline Diastolic blood pressure $(\mathrm{mmHg})$ & $77.24 \pm 7.61$ & $76.24 \pm 8.70$ & 0.64 & $82.17 \pm 9.22$ & $80.56 \pm 7.45$ & 0.40 & 0.10 \\
\hline Smoking status past/present/never & $0 / 6 / 11$ & NA & NA & $0 / 4 / 14$ & NA & NA & 0.39 \\
\hline Diabetes mellitus & 0 & NA & NA & 3 & NA & NA & 0.08 \\
\hline History of IHD & 0 & NA & NA & 0 & NA & NA & NA \\
\hline Dyslipidaemia & 3 & NA & NA & 10 & NA & NA & 0.02 \\
\hline Haemoglobin (g/L) & $134.29 \pm 11.86$ & $138.71 \pm 9.31$ & 0.02 & $135.33 \pm 18.01$ & $135.72 \pm 15.11$ & 0.85 & 0.84 \\
\hline Albumin $(g / L)$ & $39.35 \pm 2.47$ & $40.12 \pm 2.32$ & 0.19 & $38.11 \pm 2.72$ & $38.83 \pm 2.96$ & 0.34 & 0.17 \\
\hline Urea $(\mathrm{mmol} / \mathrm{L})$ & $4.26 \pm 0.95$ & $4.60 \pm 1.11$ & 0.16 & $7.31 \pm 2.15$ & $7.83 \pm 2.67$ & 0.05 & $<0.01$ \\
\hline Creatinine (mcmol/L) & $69.65 \pm 16.63$ & $69.65 \pm 15.66$ & 1.00 & $104.39 \pm 33.68$ & $108.94 \pm 31.53$ & 0.14 & $<0.01$ \\
\hline $\mathrm{eGFR}\left(\mathrm{mL} / \mathrm{min} / 1.73 \mathrm{~m}^{2}\right)$ & $97.59 \pm 15.59$ & $97.29 \pm 15.55$ & 0.83 & $67.61 \pm 20.25$ & $61.56 \pm 19.70$ & 0.02 & $<0.01$ \\
\hline Total cholesterol (mmol/L) & $5.14 \pm 1.13$ & $5.34 \pm 1.21$ & 0.16 & $4.54 \pm 0.96$ & $4.84 \pm 1.45$ & 0.30 & 0.10 \\
\hline $\mathrm{HDL}(\mathrm{mmol} / \mathrm{L})$ & $1.71 \pm 0.56$ & $1.75 \pm 0.62$ & 0.51 & $1.69 \pm 0.55$ & $1.73 \pm 0.58$ & 0.30 & 0.92 \\
\hline LDL (mmol/L) & $3.24 \pm 1.71$ & $3.07 \pm 0.89$ & 0.63 & $2.29 \pm 0.85$ & $2.56 \pm 1.25$ & 0.29 & 0.04 \\
\hline Total cholesterol/HDL & $3.29 \pm 1.27$ & $3.32 \pm 1.26$ & 0.78 & $3.00 \pm 1.28$ & $3.03 \pm 1.18$ & 0.84 & 0.50 \\
\hline Non-HDL (mmol/L) & $3.44 \pm 1.04$ & $3.60 \pm 1.04$ & 0.09 & $2.85 \pm 1.06$ & $3.11 \pm 1.37$ & 0.34 & 0.11 \\
\hline Triglyceride (mmol/L) & $1.05 \pm 0.58$ & $1.05 \pm 0.70$ & 0.97 & $1.24 \pm 0.72$ & $1.32 \pm 0.74$ & 0.55 & 0.56 \\
\hline Glucose (mmol/L) & $4.71 \pm 0.39$ & $4.82 \pm 0.40$ & 0.35 & $5.74 \pm 1.03$ & $5.69 \pm 1.34$ & 0.80 & $<0.01$ \\
\hline Corrected calcium (mmol/L) & $2.35 \pm 0.05$ & $2.39 \pm 0.07$ & 0.03 & $2.46 \pm 0.08$ & $2.46 \pm 0.07$ & 0.86 & $<0.01$ \\
\hline Inorganic phosphate (mmol/L) & $1.11 \pm 0.13$ & $1.17 \pm 0.14$ & 0.05 & $0.99 \pm 0.19$ & $1.04 \pm 0.19$ & 0.14 & 0.03 \\
\hline PTH (pmol/L) & $4.98 \pm 1.57$ & $4.53 \pm 1.09$ & 0.19 & $8.38 \pm 3.68$ & $9.42 \pm 6.46$ & 0.31 & $<0.01$ \\
\hline Vitamin D (nmol/L) & $43.65 \pm 22.03$ & $67.41 \pm 30.41$ & $<0.01$ & $57.56 \pm 25.21$ & $66.50 \pm 27.04$ & 0.04 & 0.09 \\
\hline Troponin T (ng/L) & $2.35 \pm 1.46$ & $2.76 \pm 1.72$ & 0.13 & $8.33 \pm 4.94$ & $8.39 \pm 4.85$ & 0.91 & $<0.01$ \\
\hline NT-pro-BNP (ng/L) & $43.65 \pm 25.57$ & $40.41 \pm 40.53$ & 0.62 & $190.72 \pm 172.10$ & $189.44 \pm 181.07$ & 0.96 & $<0.01$ \\
\hline hsCRP (mgl/L) & $1.90 \pm 3.51$ & $1.19 \pm 1.64$ & 0.45 & $2.58 \pm 2.95$ & $1.66 \pm 1.68$ & 0.19 & 0.54 \\
\hline Iron (mcmol/L) & $18.75 \pm 6.40$ & $21.56 \pm 7.72$ & 0.06 & $15.50 \pm 5.87$ & $17.44 \pm 6.12$ & 0.26 & 0.20 \\
\hline Transferrin (g/L) & $2.82 \pm 0.32$ & $2.83 \pm 0.36$ & 0.78 & $2.18 \pm 0.32$ & $2.25 \pm 0.37$ & 0.17 & 0.23 \\
\hline Ferritin (mcmol/L) & $82.69 \pm 78.98$ & $87.69 \pm 73.04$ & 0.55 & $452.61 \pm 1179.15$ & $291.72 \pm 579.02$ & 0.28 & $<0.01$ \\
\hline Transferrin saturation (\%) & $27.50 \pm 11.27$ & $31.19 \pm 12.58$ & 0.08 & $29.44 \pm 13.17$ & $31.61 \pm 11.37$ & 0.48 & 0.54 \\
\hline ACR (mg/mmol) & $0.49 \pm 1.35$ & $0.34 \pm 0.78$ & 0.76 & $5.53 \pm 5.46$ & $5.58 \pm 7.20$ & 0.97 & $<0.01$ \\
\hline PCR (mg/mmol) & $11.12 \pm 5.13$ & $11.35 \pm 3.82$ & 0.87 & $15.58 \pm 8.89$ & $17.20 \pm 10.83$ & 0.36 & 0.05 \\
\hline
\end{tabular}

Legend: $n$ number of participants, NA not applicable, $M / F$ male/female, IHD ischaemic heart disease, eGFR estimated glomerular filtration rate, $H D L$ high-density lipoprotein, $L D L$ low-density lipoprotein, $P T H$ parathyroid hormone, NT-pro-BNP N-terminal-pro-brain natriuretic peptide, hsCRP highly sensitive C-reactive protein, $A C R$ albumin to creatinine ratio, $P C R$ protein to creatinine ratio

Data presented as mean \pm standard deviation

smoking $(r=0.07, P=0.79)$. Changes in FMD were not associated with any other lab values in Table $1(P>$ 0.05). Changes in PWV was associated with baseline transferrin $(r=0.52, P=0.03)$. Changes in cf-PWV were not associated with age $(r=0.18 ; P=0.49)$, BMI $(r=0.27$; $P=0.29)$, WHR $(r=0.14 ; P=0.57)$, gender $\left(\mathrm{r}_{\mathrm{pb}}=0.16\right.$;
$P=0.54), \mathrm{DM}\left(\mathrm{r}_{\mathrm{pb}}=-0.14 ; P=0.59\right)$, SBP $(r=0.30 ; P=$ $0.08)$, or DBP $(r=0.08 ; P=0.64)$. Changes in cf-PWV were not associated with any other lab values in Table 1 $(P>0.05)$. Changes in CCA-IMT was associated with baseline phosphate $(r=0.67, P<0.01)$. Changes in CCAIMT were not associated with age $(r=-0.10 ; P=0.71)$, 
Table 2 Changes in cardiovascular structure and function from baseline to second visit

\begin{tabular}{|c|c|c|c|c|c|c|c|}
\hline \multirow[t]{2}{*}{ Parameter } & \multicolumn{3}{|c|}{ Healthy controls $(n=17)$} & \multicolumn{3}{|c|}{ Kidney transplant patients $(n=18)$} & \multirow{2}{*}{$\begin{array}{l}\text { Between-group comparison } \\
\text { Baseline results } P \text {-value }\end{array}$} \\
\hline & Baseline & Second visit & $P$-value & Baseline & Second visit & $P$-value & \\
\hline Brachial FMD (\%) & $4.63 \pm 3.02$ & $3.51 \pm 2.73$ & 0.33 & $4.34 \pm 3.45$ & $2.82 \pm 2.18$ & 0.03 & 0.79 \\
\hline Brachial NMD (\%) & $16.00 \pm 5.47$ & $17.17 \pm 5.18$ & 0.31 & $15.15 \pm 6.08$ & $15.74 \pm 3.88$ & 0.68 & 0.67 \\
\hline Cf-PWV (m/s) & $6.96 \pm 1.26$ & $7.17 \pm 1.50$ & 0.51 & $7.83 \pm 1.76$ & $8.44 \pm 2.28$ & 0.03 & 0.10 \\
\hline Mean ABPI & $1.18 \pm 0.08$ & $1.21 \pm 0.11$ & 0.43 & $1.27 \pm 0.15$ & $1.23 \pm 0.14$ & 0.34 & 0.47 \\
\hline Mean CCA-IMT (mm) & $5.54 \pm 1.08$ & $5.73 \pm 1.34$ & 0.22 & $5.73 \pm 0.95$ & $6.07 \pm 0.98$ & 0.02 & 0.05 \\
\hline
\end{tabular}

Legend: $n$ number of participants, FMD flow-mediated dilation, NMD nitroglycerin-mediated dilation, Cf-PWV carotid-femoral pulse wave velocity, $A B P I$ anklebrachial pressure index, CCA-IMT common carotid intima-media thickness Data presented as mean \pm SD

BMI $(r=-0.08 ; P=0.78)$, WHR $(r=-0.29 ; P=0.26)$, gender $\left(\mathrm{r}_{\mathrm{pb}}=0.14 ; P=0.59\right), \mathrm{DM}\left(\mathrm{r}_{\mathrm{pb}}=-0.25 ; P=0.34\right)$, SBP $(r=0.23 ; P=0.19)$, or DBP $(r=0.04 ; P=0.81)$. Changes in CCA-IMT were not associated with any other lab values in Table $1(P>0.05)$.

\section{Discussion}

This study shows the worsening of vascular structure and function in stable kidney transplant patients, where FMD decreased while cf-PWV and CCA-IMT increased. Change in eGFR was not associated with changes in FMD, CCA-IMT, or cf-PWV. Traditional risk factors including age, BMI, WHR, gender, DM, SBP, and DBP did not correlate with the changes seen. Baseline haemoglobin, corrected calcium, and transferrin was associated with changes in FMD. Baseline transferrin was associated with changes in cf-PWV while baseline phosphate was associated with changes in CCA-IMT.

CKD patients exhibit endothelial dysfunction as measured by FMD $[3,5]$. In previous studies, transplantation has been shown to improve FMD, acutely and at 12 months $[14,15]$. This recovery may be due to improvements in traditional and uraemia-related non-traditional risk factors [6]. Despite this, FMD values were often still lower compared to controls $[5,6]$.

CKD patients demonstrate accelerated atherosclerosis as evidenced by high CCA-IMT [3, 6]. The impact of renal transplantation on CCA-IMT is conflicting. One study demonstrated CCA-IMT to progressively increase after 2, 4, and 6 months post-transplantation [16]. Another reported improvements 6 months after transplant [17]. Despite this, values are often still higher compared to the general population $[3,17]$.

Cf-PWV is a marker of arterial stiffness and predicts the appearance of CVD in CKD, including in transplant patients $[11,18,19]$. Studies evaluating the progression of arterial stiffness over time is conflicting in transplant patients. One study reported no significant change at 12 months after transplantation, while another reported an improvement $[19,20]$. Bachelet-Rousseau's group compared cf-PWV progression in transplant waitlisted patients who were eventually transplanted or were still transplant-pending. No difference in cf-PWV was observed at baseline and upon 1-year follow-up with a short median time of $6.3(3.8-10.1)$ months posttransplantation [21]. In contrast, Strozecki's group showed cf-PWV to progress in transplant patients who were enrolled much later at $36 \pm 27$ months posttransplantation [22].

Most studies evaluating changes in FMD, CCA-IMT, and cf-PWV in transplant patients do so in immediately post-transplanted subjects. Improvements or nonprogression in these parameters shortly after transplantation does not exclude a reversal in recovery. Unlike these studies, our patients were recruited long into the post-transplant period at a median of 86 months posttransplantation. Different pathophysiological mechanisms exist in the development of endothelial dysfunction, accelerated atherosclerosis, and arterial stiffness. Successful kidney transplantation can eliminate important factors that contribute to the progression of these vascular aberrations. However, during long-term followup, cardiovascular risk factors often remain and some even worsen, which may explain the progression seen. Additionally, immunosuppression may prevent further improvement and eventual deterioration.

Failure in graft function can explain the progression of endothelial dysfunction in the late post-transplant period. Elimination of ureamic milieu may be particularly important in the restoration of vascular structure and function immediately after transplantation. Interestingly, we found baseline corrected calcium to be associated with changes in FMD and baseline phosphate to be associated with changes in CCA-IMT. Studies have described progressively decreasing FMD with greater renal impairment $[3,5]$. Improvements in eGFR after transplantation can explain improvements in FMD in the newly transplanted. However, continued functional decline after kidney transplantation has been noted, studies have reported rates of graft function loss ranging from $-1.90 \mathrm{~mL} / \mathrm{min} / \mathrm{y}$ to $-2.38 \mathrm{~mL} / \mathrm{min} / \mathrm{y}$ in transplant recipients [23-25]. Indeed, our transplant patients demonstrated worsening kidney function at their 
follow-up visit. However, we could not demonstrate an association between the decline in eGFR and FMD upon follow-up. We too did not demonstrate an association between changes in cf-PWV and eGFR. However, in contrast, associations between PWV and eGFR are inconclusive [26, 27]. eGFR has also been shown to be a significant independent predictor of CCA-IMT [28]. Hence, the restoration in eGFR post-transplant can explain improvements in CCA-IMT. In a study by Yilmaz et al., the improvement in CCA-IMT in their cohort of 178 newly transplanted patients was associated with an accompanying increase in eGFR [17]. Nevertheless, the decline in graft function thereafter can also explain the inevitable progression of CCA-IMT. Yet, again, although we observed parallel changes in eGFR and CCA-IMT in our transplant patients, we did not demonstrate an association between the two. However, consider that the mean eGFR increased from $6.3 \pm 4.0 \mathrm{~mL} / \mathrm{min} / 1.73 \mathrm{~m}^{2}$ to $85.8 \pm 13.7 \mathrm{~mL} / \mathrm{min} / 1.73$ $\mathrm{m}^{2}$ in patients from Yilmaz' group [17]. Whereas we only observed a decrease of $6.06 \pm 9.60 \mathrm{~mL} / \mathrm{min} / 1.73$ $\mathrm{m}^{2}$ upon follow-up. Correlations between CCA-IMT and eGFR may only be seen with substantial changes in eGFR.

It is important to consider the role of immunosuppressants in enabling the progression seen. Calcineurin inhibitors increase endothelin levels and abrogate nitric oxide-induced vasodilation, making them potent vasoconstrictors [29, 30]. Additionally, they promote intravascular fibrosis and cause sodium retention leading to hypertension [30]. Hence, these unwanted effects from immunosuppressants may impair endothelial function and promote arterial stiffness in the long run $[5,6]$.

Another factor that warrants consideration is the dyslipidaemia seen in our transplant patients. Metabolic syndrome is a novel multiplex CVD risk factor that includes dyslipidaemia, central obesity, dysglycaemia, and hypertension. It is common in those with CKD, evidenced by reports of prevalence ranging from $30 \%$ in stage 3-4 CKD to $69 \%$ in incident haemodialysis patients [31, 32]. Our transplant population had more dyslipidaemics compared to controls. In a cohort of 198 CKD patients, CCA-IMT was found to be closely associated with traditional cardiovascular risk factors, including dyslipidaemia and DM [33].

Anaemia is common in CKD and its aetiology is multifactorial in nature. However, erythropoietin deficiency seems to be the major factor for explaining low haemoglobin in CKD patients. We found that baseline haemoglobin predicted change in FMD in our transplant patients, while baseline transferrin predicted change in FMD and cf-PWV. Indeed, previously it has been reported that haemoglobin is inversely related to FMD in
CKD patients with or without diabetes [34, 35]. In contrast, the specific effect of transferrin on PWV and FMD in CKD or kidney transplant patients has not been extensively investigated in the literature.

Ultimately, our study had a small sample size, hence it was difficult to reveal correlations between traditional and non-traditional risk factors with the changes observed, including uraemia, and aspects of metabolic syndrome.

\section{Conclusion}

Our study demonstrates that progressive worsening in surrogate markers of vascular structure and function occur in the post-transplant period upon long-term follow-up. Although transplantation initially alleviates the cardiovascular burden, the vascular disease progresses in the long-term beyond the initial 'honeymoon' period. Since cardiovascular mortality is the commonest cause of mortality amongst transplant patients, the nephrology community has long sought for interventions that could improve the adverse cardiovascular picture in such patients. Thus, it is important to further characterise the nature of the changes in vascular abnormalities in kidney transplant recipients, which this pilot study achieves. The ultimate goal of this study is to determine the power needed to demonstrate a difference with an intervention on FMD, cf-PWV, and CCA-IMT in kidney transplant patients.

\section{Abbreviations}

ABPI: Ankle-brachial pressure index; BMI: Body mass index; BP: Blood pressure; CCA-IMT: Common carotid artery intima-media thickness; cfPWV: Carotid-femoral pulse wave velocity; CKD: Chronic kidney disease; CKDEPI: Chronic kidney disease epidemiology collaboration; CVD: Cardiovascular disease; eGFR: Estimated glomerular filtration rate; FMD: Brachial artery flowmediated dilation; IQR: Interquartile range; WHR: Waist to hip ratio

\section{Acknowledgements}

All patients who participated.

\section{Authors' contributions}

DB design, data collection, manuscript, supervision. JC design, manuscript, supervision. NH data collection, data interpretation, manuscript. JJ data collection, analysis, manuscript. RL-J data collection, data interpretation, manuscript. RR data collection, data interpretation, manuscript. All authors have read and approved the manuscript.

\section{Funding}

The study was partially funded by Wellcome ISSF 204809/Z/16/. They had no role in the design of the study nor the collection, analysis, and interpretation of data and writing of the manuscript.

\section{Availability of data and materials}

The datasets used and/or analysed during the current study are de-identified and available from the corresponding author on reasonable request.

\section{Ethics approval and consent to participate}

All procedures performed in studies involving human participants were in accordance with the ethical standards of the institutional and/or national research committee and with the 1964 Helsinki declaration and its later amendments or comparable ethical standards. The study was submitted to and approved by the London South East Research Ethics Committee. This 
article does not contain any studies with animals performed by any of the authors. Informed written consent was obtained from all individual participants included in the study.

\section{Consent for publication}

Not applicable.

\section{Competing interests}

DB has received Grants from British Heart Foundation PG 10/71/28462; DB has received honorarium from AstraZeneca, Vifor Pharma, and Pfizer. JJ, NH, $R R, R L-J$, and JK have no conflict of interest.

Received: 12 August 2019 Accepted: 12 December 2019

Published online: 08 January 2020

\section{References}

1. Santoro A, Mandreoli M. Chronic renal disease and risk of cardiovascular morbidity-mortality. Kidney Blood Press Res. 2014;39:142-6.

2. Di Lullo L, House A, Gorini A, et al. Chronic kidney disease and cardiovascular complications. Heart Fail Rev. 2015;20:259-72.

3. Recio-Mayoral A, Banerjee D, Streather C, et al. Endothelial dysfunction, inflammation and atherosclerosis in chronic kidney disease--a crosssectional study of predialysis, dialysis and kidney-transplantation patients. Atherosclerosis. 2011:216:446-51.

4. Ojo A, Hanson J, Wolfe R, et al. Long-term survival in renal transplant recipients with graft function. Kidney Int. 2000:57:307-13.

5. Sharma J, Kapoor A, Muthu R, et al. Assessment of endothelial dysfunction in asian indian patients with chronic kidney disease and changes following renal transplantation. Clin Transpl. 2014;28:889-96.

6. Yildiz G, Duman A, Aydin $\mathrm{H}$, et al. Evaluation of association between atherogenic index of plasma and intima-media thickness of the carotid artery for subclinic atherosclerosis in patients on maintenance hemodialysis. Hemodial Int. 2013;17:397-405.

7. Gimbrone MA Jr, Garcia-Cardena G. Endothelial cell dysfunction and the pathobiology of atherosclerosis. Circ Res. 2015;118:620-36.

8. Moody WE, Edwards NC, Madhani M, et al. Endothelial dysfunction and cardiovascular disease in early-stage chronic kidney disease: cause or association? Atherosclerosis. 2013;223:86-94.

9. Otsuka F, Sakakura K, Yahagi K, et al. Has our understanding of calcification in human coronary atherosclerosis progressed? Arterioscler Thromb Vasc Biol. 2013;34:724-36.

10. Chitalia N, Ismail T, Tooth $L$, et al. Impact of vitamin D supplementation on arterial vasomotion, stiffness and endothelial biomarkers in chronic kidney disease patients. PLoS One. 2014;9(3):e91363.

11. Dahle DO, Eide IA, Asberg A, et al. Aortic stiffness in a mortality risk calculator for kidney transplant recipients. Transplantation. 2015;99:1730-7.

12. Sidhu JS, Newey VR, Nassiri DK, et al. A rapid and reproducible on line automated technique to determine endothelial function. Heart. 2002;88(3): 289-92.

13. Levey AS, Stevens LA, et al. A new equation to estimate glomerular filtration rate. Ann Intern Med. 2009;150:604-12.

14. Kocak H, Ceken K, Yavuz A, et al. Effect of renal transplantation on endothelial function in haemodialysis patients. Nephrol Dial Transplant. 2006;21:203-7.

15. Oflaz $\mathrm{H}$, Turkmen A, Turgut $\mathrm{F}$, et al. Changes in endothelial function before and after renal transplantation. Transpl Int. 2006;19:333-7.

16. Nafar M, Khatami F, Kardavani B, et al. Atherosclerosis after kidney transplantation: changes of intima-media thickness of carotids during early posttransplant period. Urol J. 2006:4:105-10.

17. Yilmaz MI, Sonmez A, Saglam M, et al. A longitudinal study of inflammation, CKD-mineral bone disorder, and carotid atherosclerosis after renal transplantation. Clin J Am Soc Nephrol. 2015;10:471-9.

18. Baumann M, Wassertheurer S, Suttmann $Y$, et al. Aortic pulse wave velocity predicts mortality in chronic kidney disease stages 2-4. J Hypertens. 2014;32: 899-903.

19. Birdwell KA, Jaffe $G$, Bian A, et al. Assessment of arterial stiffness using pulse wave velocity in tacrolimus users the first year post kidney transplantation. A prospective cohort study. BMC Nephrol. 2015;16:93. https://doi.org/10. 1186/s12882-015-0092-7.

20. Zoungas $S$, Kerr PG, Chadban $S$, et al. Arterial function after successful renal transplantation. Kidney Int. 2004;65:1882-9.
21. Bachelet-Rousseau C, Kearney-Schwartz A, Frimat L, et al. Evolution of arterial stiffness after kidney transplantation. Nephrol Dial Transplant. 2011; 26:3386-91.

22. Strozecki P, Adamowicz A, Kozlowski M, et al. Progressive arterial stiffening in kidney transplant recipients. Ann Transplant. 2011;16:30-5.

23. Samaan F, Requiao-Moura LR, Pinheiro HS, et al. Prevalence and progression of chronic kidney disease after renal transplantation. Transplant Proc. 2011; 43:2587-91.

24. Kukla A, Adulla M, Pascual J, et al. CKD stage-to-stage progression in native and transplant kidney disease. Nephrol Dial Transplant. 2008;23:693-700.

25. Djamali A, Kendziorski C, Brazy PC, et al. Disease progression and outcomes in chronic kidney disease and renal transplantation. Kidney Int. 2003;64:1800-7.

26. Townsend RR, Wimmer NJ, Chirinos JA, et al. Aortic PWV in chronic kidney disease: a CRIC ancillary study. Am J Hypertens. 2010;23:282-9.

27. Hermans MM, Henry R, Dekker JM, et al. Estimated glomerular filtration rate and urinary albumin excretion are independently associated with greater arterial stiffness. The Hoorn study. J Am Soc Nephrol. 2007:18:1942-52.

28. Kawamoto R, Ohtsuka N, Kusunoki T, et al. An association between the estimated glomerular filtration rate and carotid atherosclerosis. Intern Med. 2008:47:391-8.

29. Seibert FS, Steltzer J, Melilli E, et al. Differential impact of belatacept and cyclosporine a on central aortic blood pressure and arterial stiffness after renal transplantation. Clin Transpl. 2014;28:1004-9.

30. Szymczak M, Kluz J, Malecki R, et al. Effect of immunosuppressive treatment on carotid atherosclerosis in renal transplant recipients. Transplant Proc. 2016;48:1626-9.

31. Johnson DW, Armstrong K, Campbell SB, et al. Metabolic syndrome in severe chronic kidney disease: prevalence, predictors, prognostic significance and effects of risk factor modification. Nephrology (Carlton). 2007;12:391-8.

32. Young DO, Lund RJ, Haynatzki G, et al. Prevalence of the metabolic syndrome in an incident dialysis population. Hemodial Int. 2007;11:86-95.

33. Hinderliter A, Padilla RL, Gillespie BW, et al. Association of carotid intimamedia thickness with cardiovascular risk factors and patient outcomes in advanced chronic kidney disease: the RRI-CKD study. Clin Nephrol. 2015;84:10-20.

34. Sonmez A, Yilmaz MI, Saglam M, et al. The relationship between hemoglobin levels and endothelial functions in diabetes mellitus. Clin J Am Soc Nephrol. 2010;5(1):45-50

35. Yilmaz MI, Sonmez A, Saglam M, et al. Hemoglobin is inversely related to flow-mediated dilatation in chronic kidney disease. Kidney Int. 2009;75(12): 1316-21.

\section{Publisher's Note}

Springer Nature remains neutral with regard to jurisdictional claims in published maps and institutional affiliations.
Ready to submit your research? Choose BMC and benefit from:

- fast, convenient online submission

- thorough peer review by experienced researchers in your field

- rapid publication on acceptance

- support for research data, including large and complex data types

- gold Open Access which fosters wider collaboration and increased citations

- maximum visibility for your research: over $100 \mathrm{M}$ website views per year

At $\mathrm{BMC}$, research is always in progress.

Learn more biomedcentral.com/submissions 